\title{
The Yeasts
}

Teun Boekhout ${ }^{1,2}{ }_{2}$ Heide-Marie Daniel ${ }^{3}$, Marizeth Groenewald ${ }^{1}$, Andrey Yurkov ${ }^{4}$, Feng-Yan Bai ${ }^{5}$, Vincent Robert ${ }^{1}$ ${ }^{1}$ Westerdijk Fungal Biodiversity Institute, Utrecht, ${ }^{2}$ Institute of Biodiversity and Ecosystem Dynamics, University of Amsterdam, The Netherlands; ${ }^{3} \mathrm{BCCM} / \mathrm{MUCL}$, Earth and Life Institute, Université catholique de Louvain, Louvain-la-Neuve, Belgium; “4eibniz Institute DSMZ - German Collection of Microorganisms and Cell Cultures, Braunschweig, Germany; Institute of Microbiology, Chinese Academy of Sciences, Beijing, China Contact: t.boekhout@westerdijkinstitute.nl

Introduction

Yeast diversity is rapidly expanding due to discovery of new species and new insight in their phylogenetic relationships. This also relates to emerging yeasts that are clinically relevant. THE YEASTS aims to collect and to present information on all yeast species in an updatable and online, open access electronic format.

Until 2011, information on yeasts diversity and taxonomy was published in a book series, called 'The Yeasts, a Taxonomic Study' that was published by Elsevier. A major disadvantage of such a book series is that regular updates cannot be made easily.

The 5th edition of 'The Yeasts, a Taxonomic Study' was outdated soon after publication in 2011 as the field of yeast diversity and taxonomy is rapidly developing, due to

1. Fast and ongoing species discovery due to the use of molecular barcoding;

2. The application of molecular phylogenies that caused (and will cause) many taxonomic changes; and

3. Implementation of the 'One Fungus = One Name' principle.

Species description rates are faster than ever

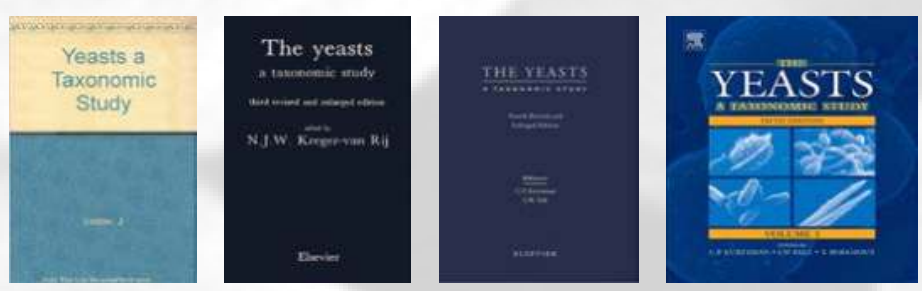

THE YEASTS is an OPEN SCIENCE project that will be available on-line allowing regular updates.

The 'FOUNDATION THE YEASTS' (KvK 71086544) offers a legal frame work to further develop and maintain this project.

\section{Methods}

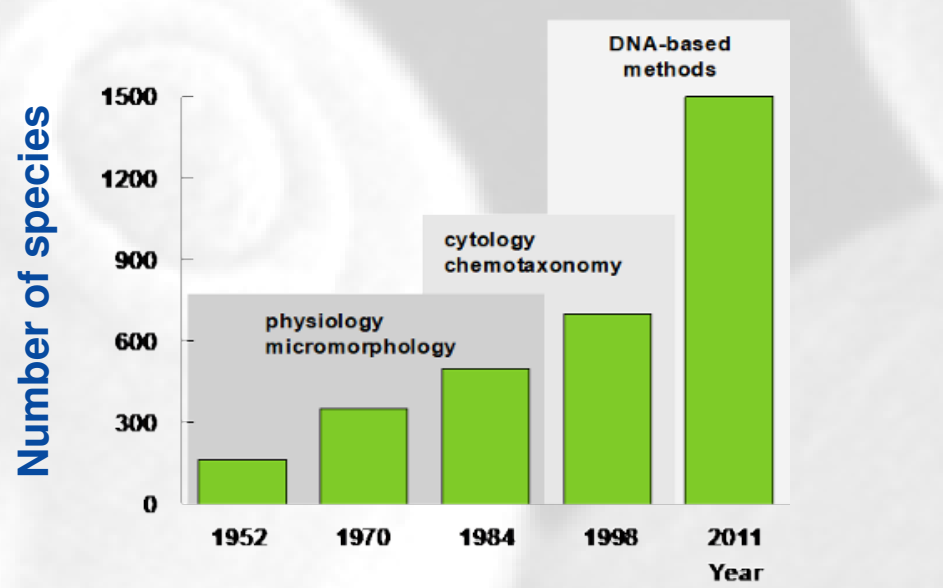

Public information on authenticated yeast strains from public culture collections and other sources will be made available in a species-based electronic information platform using the Biolomics bio-informatics pipeline (BioAware). Data obtained from strains will be clustered in species, and this species database will be used to prepare species and genus descriptions. Information on yeast strains will be clustered according to genus and species boundaries, and posted at the website www.theyeasts.org.

Curation will be done by the scientific community to stay up-to-date. Protocols for the isolation and identification of yeast isolates will be provided.

\section{Results}

Taxonomic descriptions of species and genus descriptions will include data on

Morphology, asexual and sexual reproduction

Carbon and nitrogen growth requirements

Other growth tests (e.g. temperatures), biochemical tests (urease, DBB)

Reference DNA-barcodes and genomes, MALDI-TOF MS spectra

Strain origin, ecology, use in agriculture, food science and biotechnology

Clinical relevance

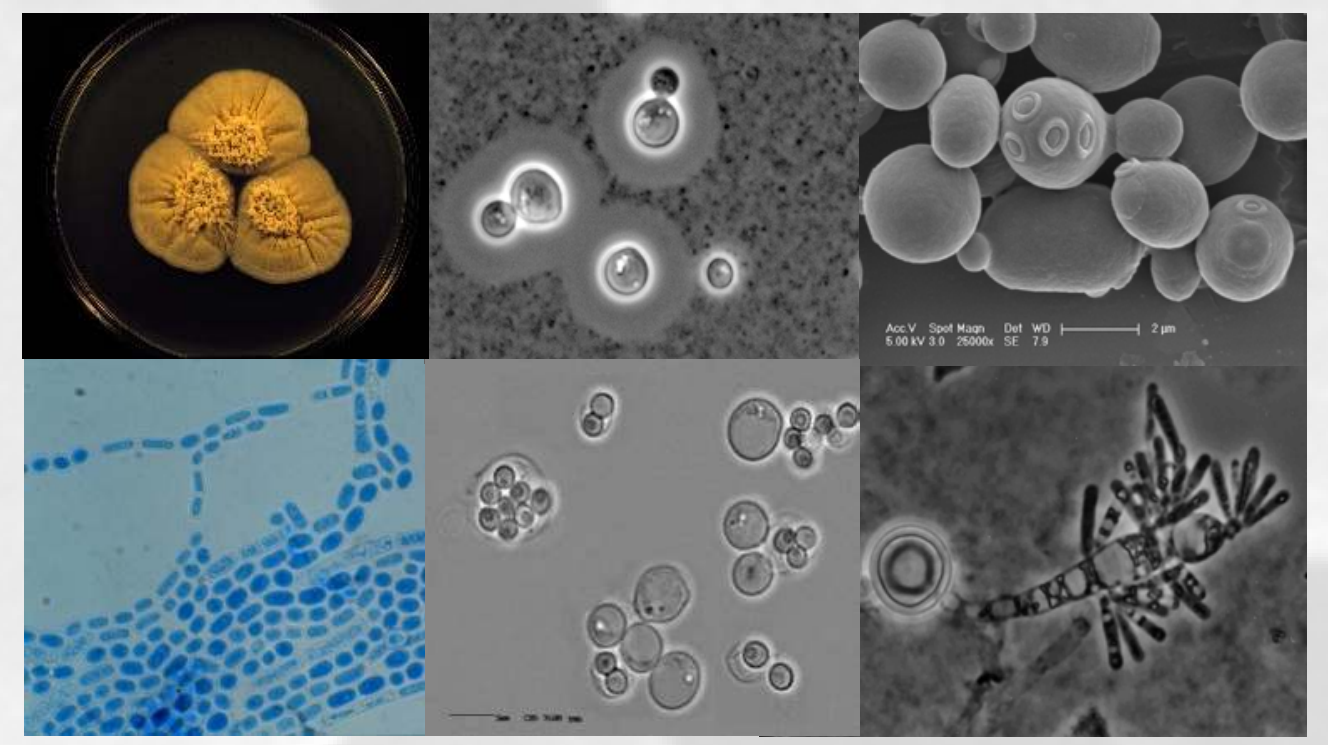

Illustrations of colonies, and microscopic structures of cells will be included. Linda Barnett, the widow of the late Dr. James Barnett, made her collection of high quality yeast micrographs available for the project.

Molecular barcodes, nutritional growth data, and MALDI-TOF spectra will be used strain identification. Sequence data of resistance-related genes, e.g. ERG11 of Candida albicans and FKS1 and FKS2 of C. glabrata, will be included to identify resistant isolates.

\section{Discussion and outlook}

THE YEASTS is a highly useful resource for clinicians and laboratory personnel dealing with the identification of yeast isolates obtained from clinical environments, including so-called non-conventional yeasts.

We aim to intensify collaboration between THE YEASTS and the ISHAM communities on e.g. yeasts susceptibility data, resistance genes, and clinical background, to further increase the usefulness of THE YEASTS for clinicians.

THE YEASTS is endorsed by the International Commission of Yeasts (ICY), part of International Union of Microbiological Societies (IUMS) and is financially supported by Health Holland, part of Topsector Life Sciences \& Health, The Netherlands (LSHM 18003-H003) 